\title{
Fast-Track Cardiac Anaesthesia at a Tertiary Care Cardiac Centre in Nepal
}

\author{
Jeju Nath Pokharel*, Apurba Sharma, Murari Raj Upreti, Raamesh Koirala, \\ Jyotindra Sharma, Siddartha Pradhan \\ Shahid Gangalal National Heart Centre, Bansbari, Kathmandu, Nepal
}

\section{Citation \\ Pokharel JN, Sharma A, Upreti MR, et al. Fast- Track Cardiac Anaesthesia at a Teritory Care Cardiac Centre in Nepal. Nepalese Heart Journal 2013;10(1):6-11}

Keywords

Anaesthesia, cardiac, fast-track

\begin{abstract}
Background: With the evolution of anesthesia and surgical procedures, fast track cardiac anaesthesia has gained an increased interest, mainly based on the possibility of reducing health costs without compromising patient care and has been implemented at a tertiary care cardiac centre in Nepal.
\end{abstract}

Objective: The purpose of this study is to evaluate the status of fast track cardiac anaesthesia after open heart procedures and suitability of existing anaesthetic technique for fast-track extubation.

Methods: After standard anaesthesia and surgical technique, we retrospectively reviewed the duration of mechanical ventilation, length of Intensive Care Unit stay, reintubation, and incidence of inhospital mortality in consecutive adult patients undergoing open heart surgery during six months period.

Results: The median time of the extubation was six hours, median days of the ICU stay was three days. Five cases were reintubated because of the respiratory failure which is about $1.8 \%$ out of 277 cases. Seven patients (2.5\%) died of sepsis and low cardiac output syndrome.

Conclusions: Fast track anaesthesia is suitable with applied anesthetic technique in our centre. However there exists relatively small risk of re-intubation (1.8\%).

In this study we retrospectively reviewed the time to extubation and suitability of existing anaesthetic technique for fast-track extubation in patients undergoing cardiac surgical procedures under cardiopulmonary bypass in a tertiary care cardiac centre.

\footnotetext{
*Corresponding Author:

Jeju Nath Pokhrel

Shahid Gangalal National Heart Centre,

Bansbari, Kathmandu, Nepal

Email: pjejunath@yahoo.com
} 


\section{METHODS}

The study was designed as a retrospective observational study. Eligible patients were consecutive elective adult patients who underwent open heart surgery during six months period from January 2010 to June 2010 at the institution.

\section{Anesthesia Technique}

All the patients reviewed in the study received FTCA. Premedication consisted of oral diazepam 5 to $10 \mathrm{mg}$ given at night before and two to four hours before surgery. In the operating room monitoring of ECG and pulse oximetry was initiated. Under local anaesthesia and asceptic precautions, a $16-\mathrm{G}$ intravenous cannula was inserted in the cephalic or cubital vein, a $20-\mathrm{G}$ intra-arterial cannula was introduced into the radial artery for monitoring of the arterial pressure and obtaining arterial blood for analysis and central venous cannulation was done with triple lumen cannula for central venous pressure monitoring. Oxygen was administered via nasal prongs at 1 to $4 \mathrm{~L} / \mathrm{min}$ if prescribed by the attending anaesthesiologist. Anaesthesia was induced with midazolam $0.05 \mathrm{mg} / \mathrm{kg}$, fentanyl 5 to $10 \mathrm{mcg} / \mathrm{kg}$, ketamine 0.5 to $1 \mathrm{mg} / \mathrm{kg}$ and propofol $1 \mathrm{mg} / \mathrm{kg}$ intravenously. Tracheal intubation was facilitated with vecuronium 0.1 to $0.2 \mathrm{mg} / \mathrm{kg}$. Anaesthesia was maintained with infusion of propofol at the rate of $100 \mathrm{mcg} / \mathrm{kg} / \mathrm{min}$, isoflurane (end tidal concentration 0.5 to $1.5 \%$ ) and oxygen/air 50 to $70 \%$ before cardiopulmonary bypass (CPB). After initiation of $\mathrm{CPB}$ propofol infusion was continued and fentanyl 5 to $10 \mathrm{mcg} / \mathrm{kg}$ and vecuronium 0.1 to $0.2 \mathrm{mg} / \mathrm{kg}$ was added.

Standard surgical and perfusion techniques were used in all patients. Inspired and expired gas concentration of oxygen $\left(\mathrm{O}_{2}\right)$, carbon dioxide $\left(\mathrm{CO}_{2}\right)$ and isoflurane were measured using anaesthetic gas monitoring system. Haemodynamic parameters were maintained within $20 \%$ of the basal values with small boluses of IV nitroglycerine or ephedrine/phenylephrine /noradrenaline and small boluses of IV metoprolol /esmolol or atropine/epicardial pacing as required. Filling pressures and fluid balance was maintained using lactated Ringers solution, Gelofusine $\AA$, blood and blood products as necessary.

If needed, infusions of dopamine/milrinone /adrenaline/noradrenaline/nitro-glycerine were used as inotropes/vasodilators while coming off by-pass to maintain haemodynamics.

At the end of surgical procedure, patient was shifted to surgical intensive care unit (SICU). Propofol infusion was maintained at the rate of
$25-50 \mathrm{mcg} / \mathrm{kg} / \mathrm{min}$ for one to four hours in the SICU. Analgesia in the SICU was provided by intravenous morphine till extubation and with tablet paracetamol when patient started taking orally. Patient was warmed with forced air warming system (Bair Hugger Warming Unit, Arizant Healthcare Inc. MN, USA) if required.

All the patients were ventilated in synchronised mandatory mode of ventilation (SIMV) with pressure support. These ventilated patients were weaned by standard protocol when haemodynamically stable, awake, with no signs of residual neuromuscular blockade and then extubated.

Mechanical ventilation was continued with sedation if patient had excessive drains or advised by attending physician. Duration of mechanical ventilation was defined as time from skin closure to extubation of trachea.

\section{Data Collection}

Clinical data (duration of mechanical ventilation, ICU length of stay (LOS), reintubation, and incidence of inhospital mortality) for the patients were retrospectively registered in a dedicated electronic research database from the hospital records. Final data was transferred into an SPSS database, from which further analysis were performed.

\section{RESULTS}

A total of 277 patients who underwent open heart procedure were included in the study. There were 139 (50.2\%) female and 138 (49.8\%) male patients. Patient and cardiopulmonary bypass characteristics are shown in table 1.

Table1: Demographic and Cardiopulmonary Bypass characteristics:

\begin{tabular}{ll}
\hline Patient and CPB Characteristics & \\
\hline Age, years (Mean+SD) & $38.5 \pm 15.7$ \\
Sex (M/F) & $138 / 139$ \\
Weight, kg (Mean+SD) & $52.6 \pm 18.4$ \\
CPB time,min (Mean+SD) & $63.4 \pm 24.1$ \\
Cross clamp time, min (Mean+SD) & $37.2 \pm 23.8$ \\
Duration of anaesthesia, min (mean+SD) & $134.8 \pm 35.2$ \\
\hline
\end{tabular}

Almost half of the patients had valvular heart disease. Adults with congenital heart disease comprised of $23 \%$ of patients and coronary artery disease contributed $22 \%$ of all the patients. Distribution of patients with respect to their diagnosis is given in figure 1 .

CHD - congenital heart disease, VHD - valvular heart disease, $\mathrm{CAD}$ - coronary artery disease. 
One hundred and eighty-eight of 277 (67.8\%) of patients were extubated within six hours after skin closure (Figure 2). Five cases were reintubated because of the respiratory failure which is about $1.8 \%$ out of 277 cases. Among those 188, who were extubated within six hours, two patients $(1 \%)$ had to

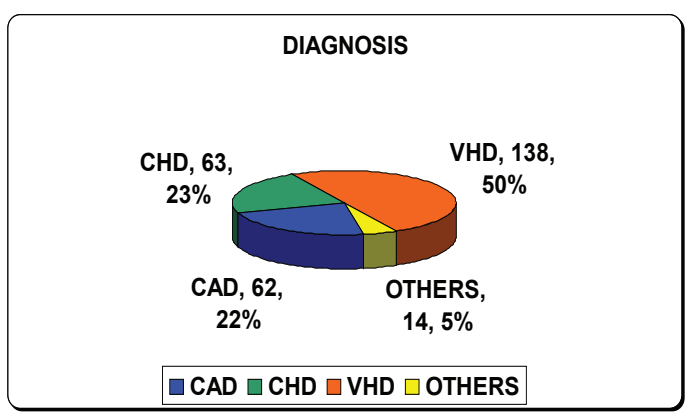

Figure 1: Distribution of adult patients with respect to different diagnoses of heart diseases:

be re-intubated later as they required mechanical ventilation after initial tracheal extubation. Three of 89 patients $(3.3 \%)$, who were extubated after six hours of mechanical ventilation, required re-intubation of trachea. Among those 188, who were extubated within six hours, one patient died, however, a higher mortality (six of $89,6.7 \%$ ) was seen among patients who required mechanical ventilation for more than six hours after skin closure.

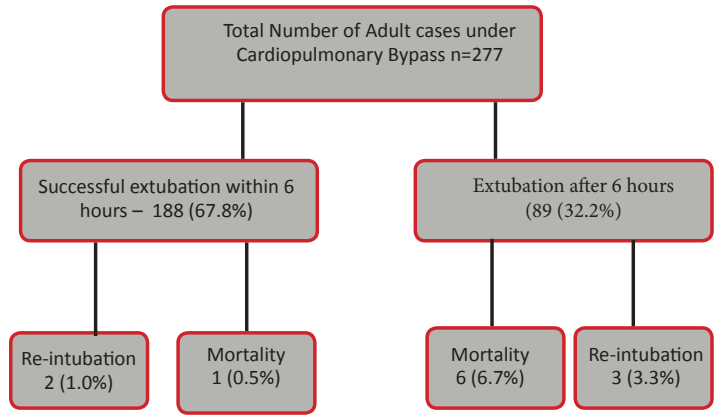

Figure 2: Flowchart showing re-intubation and mortality rate:

Overall median duration of mechanical ventilation was six hours (Figure 3). Patients with congenital heart disease (ASD, VSD) and patients who had undergone off-pump coronary artery bypass (OPCAB), coronary artery bypass graft (CABG) and single valve procedures were extubated early. Median duration of mechanical ventilation was six hours or less in such patients. But, in selected group of patients, who underwent double valve replacement (DVR), pericardiectomy, surgery for left atrial myxoma (LA Myxoma), the median duration of mechanical ventilation was more than six hours. Reason for delayed extubation was not stated in the records.

Two of $188(1.0 \%)$ patients, who were extubated within six hours, had to be re-intubated for respiratory failure. Similarly, those who were on mechanical ventilation for more than six hours, three of $89(3.3 \%)$ patients had to be re-intubated. Overall, five of 277 (1.8) patients had to be re-intubated.

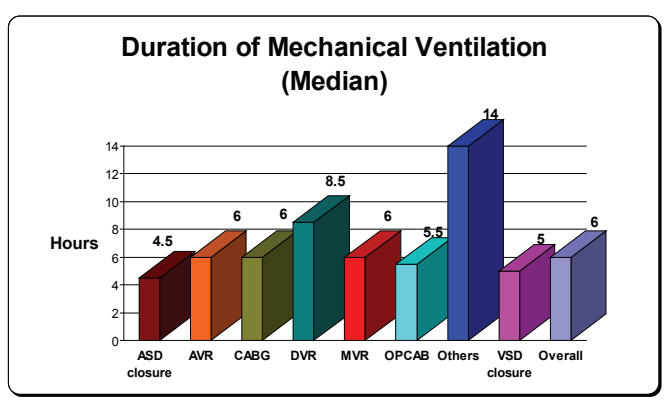

Figure 3: Figure showing duration of mechanical ventilation:

Extubation of trachea within six hours resulted in shorter ICU stay (median duration three days), whereas, delayed extubation of trachea resulted in a longer duration of ICU stay (median duration of four days). The overall median length of stay in the ICU was three days.

Mortality was higher in those who remained intubated for more than six hours (six of $89,6.7 \%$ ) than those, who were extubated within six hours (one of $188,0.5 \%$ ). Overall mortality was seven of $277(2.5 \%)$.

\section{DISCUSSION}

High-dose opioid anesthesia was introduced into cardiac surgery almost 40 years ago in an attempt to provide hemodynamic stability without myocardial depression in patients with compromised cardiac function. ${ }^{4}$ This resulted in need for a prolonged period of postoperative mechanical ventilation. However, it was accepted in the belief that it afforded a more stable and safer recovery from surgery. CABG in the elderly population in the United States has doubled every five years since 1985 and by 2003 it was estimated that 500,000 cardiac procedures were performed annually in the US at a cost of around nine billion of US dollars. ${ }^{5}$ Thus, mainly due to financial constraints, the focus of cardiac anaesthesia started shifting in the early 1990's to lower dose opioid, earlier extubation and decreased ICU stay. This came to be labeled as FTCA. ${ }^{6}$ Shorter ICU and hospital stays also mean that more operations might be performed. More operations might reduce waiting times and the number of patients who deteriorate or die while waiting for surgery. This is especially true for a developing country like Nepal, where cardiac surgical services are provided by only few institutions. The need for rapid turnover of cases 
is huge. We have developed and adopted FTCA program and is widely implemented. However, status of the implemented anesthetic technique has not been evaluated. So, this retrospective study was designed in order to identify the status of this FTCA program and to check the suitability of the anaesthetic technique for fast-track extubation.

\section{Selection of Eligible Patients}

We selected consecutive elective adult patients for a period of six months. The total number of cases during the study period was 277 . Even though, Constantinidis et $\mathrm{al}^{7}$ in 2006 reported predictors of poor postoperative outcome, no simple reliable and validated scoring system has been developed to predict fast-track eligibility with certainty, and it is recommended that FTCA eligibility should be done at the end of surgery. ${ }^{3}$ Most cardiac surgical patients should be considered for early extubation unless their intraoperative course and post-bypass status suggests otherwise $3,8,9$

\section{Safety of FTCA}

The result of our study confirms the safety of FTCA. One hundred and eighty-eight of 277 $(67.8 \%)$ patients were extubated within six hours of skin closure. Among these patients, there was only one $(0.5 \%)$ mortality. But, with the same anaesthetic technique, need for prolonged mechanical ventilation resulted in higher mortality of $6.7 \%$ (Figure 2). The need for prolonged mechanical ventilation in patients who had bleeding problems, re-exploration, haemodynamic instability, neurological complications might be the reasons for the higher mortality. However, analyses of these factors have not been done in this study as they were not documented in the records uniformly. In 2003 Myles et al ${ }^{5}$ did a meta-analysis comparing FTCA with Conventional Cardiac Anaesthesia (CCA). Overall they included 10 trials with a total of 1800 patients. They showed no statistically significant difference in mortality rate and major morbidity, markedly decreased time to tracheal extubation and decreased ICU LOS, no significant decrease in hospital LOS. They concluded that there was no evidence of increased morbidity or mortality with FTCA techniques and combined with the known cost benefits there was no reason to continue with high dose opioid techniques ${ }^{5}$. Their findings are consistent with a Cochrane Review ${ }^{10}$, also published in 2003, showed no difference between early and conventional extubation with regards to mortality in ICU or at 30 days. There was no significant difference in postoperative myocardial ischaemia, re-intubation before or after 24 hours. Early extubation also showed a decreased ICU and hospital length of stay. A meta-regression by van Mastrigt et $\mathrm{al}^{11}$ in 2006 showed that neither anaesthetic dose, nor extubation protocols were significant predictors for myocardial infarction and death following cardiac surgery. Svircevic and colleagues $^{12}$ from the Netherlands retrospectively compared FTCA and CCA using a large number of patients $(\mathrm{n}=7989)$ surpassing the two previous largest studies with $1012^{13}$ and $404^{14}$ patients respectively. The authors concluded that their results were in keeping with other studies and metaanalyses showing no effect of FTCA on mortality and morbidity.

\section{Re-intubation}

In our study the overall re-intubation rate was $1.8 \%$ and chances of re-intubation of trachea did not increase with early extubation (Figure2). In the 2003 review, Myles et al. ${ }^{5}$ reported that re-intubation was rare with a rate of usually $<2 \%$ in patients undergoing FTCA. Although one study reported a rate of $6.6 \%,{ }^{15}$ most have reported a rate of less than $1 \%^{16,17,18,19}$. Reassuringly, London and colleagues ${ }^{20}$ reported an initial rate of tracheal re-intubation for medical reasons of 7.7\%; most of these occurred beyond the initial 24 hour recovery period. After institution of a FTCA program this improved to $2.1 \%$. In the Cochrane review published in $2003^{10}$, the overall incidence of reintubation is four out of 249 patients in total $(1.6 \%)$ and is considered to be low.

\section{Methods of FTCA}

There are different fast track protocols for cardiac anaesthesia. The focus of studies has been the opioids to use and the dose, as well as the role of propofol in maintenance of anaesthesia. We have included combination of low dose propofol, ketamine, midazolam as induction agent, fentanyl as opioid and vecuronium as muscle relaxant. The most common induction agent is propofol in combination with an opioid, usually at a dose of $0.5 \mathrm{mg} / \mathrm{kg}$ with $10 \mathrm{mg}$ boluses until loss of consciousness $^{17,21,22,23,24}$ although some use propofol in higher doses ${ }^{2,5,25}$ or even start directly with an infusion. ${ }^{26}$ Etomidate, thiopentone and ketamine are described as being used. There are some centers that omit the induction agent entirely..$^{27,28}$ Fentanyl at a dose of 10 to 15 $\mu \mathrm{g} / \mathrm{kg}$ at induction is commonly used in many centers. ${ }^{2,16,17,28,24,26,30}$ Remifentanil was equally safe and effective as fentanyl with no difference in time to extubation. ${ }^{21}$

We have maintained anaesthesia with propofol, isoflurane combination in the pre by-pass period. Propofol infusion was continued during bypass and isoflurane was restarted as mechanical ventilation resumed. Propofol infusion was 
continued as sedative in the intensive care unit. Prior to a study by Myles et al ${ }^{16}$ in 1997 , propofol was a much avoided drug in cardiac anaesthesia. He compared its use in maintenance to enflurane and showed no increased mortality or awareness. There were similar haemodynamic changes with no difference in inotrope requirements. He also showed a significantly decreased time to extubation with no increase in the incidence of ischaemia or postoperative myocardial infarction. Since then propofol has been used commonly in cardiac anaesthesia, either as part of a total intravenous anaesthetic technique $e^{5,23,28}$ or as sedation on CPB and continuing into the ICU. $2,5,17,22,24,25,26$

The most common agent used for sedation of these patients post bypass and into the ICU is

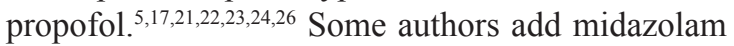
either as boluses or an infusion. ${ }^{5}$ We have continued with propofol infusion.

\section{Pain Management of the FTCA Patient}

Pain management poses a significant problem after cardiac surgery and has been one of the main criticisms of FTCA. There are many painful interventions e.g. sternotomy, drain insertion and leg vein harvesting. Inadequate analgesia increases morbidity through adverse effects on haemodynamics, metabolism, immunology and haemostasis. ${ }^{29}$ There is also the need for effective cough and deep breathing as a means to prevent atelectasis and decrease pneumonia. By hindering these, pain can be detrimental to weaning from mechanical ventilation in the post operative period. But, Chaney, in a 2006 review, states that patient satisfaction is determined as much by the comparison between anticipated and experienced pain as it is by the actual level of pain experienced. ${ }^{30}$ Thus patients, who generally expect severe pain after cardiac surgery may not complain of significant pain in the postoperative period despite moderate intensity of pain being experienced.

\section{Opioids and NSAID}

Postoperative morphine was the most common analgesic drug used in most studies, although no study was testing its use. Methods included infusions, boluses and patient controlled analgesia. We have used nurse administered intermittent intravenous bolus technique. NSAIDs have been described for postoperative analgesia in ICUs. 17,21,24,27 We have followed intravenous morphine with oral paracetamol due to its easy availability.

Limitations of the study are its retrospective nature, no analysis of complications, ICU re-admission, incidence of intraoperative awareness and cost effectiveness.

\section{CONCLUSION}

FTCA has been widely implemented with acceptable re-intubation and mortality rates. The existing anaesthetic technique is suitable for fast-track cardiac anaesthesia.

\section{REFERENCES}

1. CM Deshpande, SN Mohite, Prashant Kamdi. Sufentanil Vs Fentanyl for Fast-Track Cardiac Anaesthesia. Indian J Anaesth. 2009 August; 53(4): 455-462.

2. Reis J, Mota JC, Ponce P, Costa-Pereira A, Guerreiro M. Early extubation does not increase complication rates after coronary artery bypass graft surgery with cardiopulmonary bypass. Eur J Cardiothorac Surg 2002;21:1026-1030.

3. Cheng DC. Fast track cardiac surgery pathways: early extubation, process of care, and cost containment. Anesthesiology 1998; 88:142933.

4. Lowenstein E, Hallowell P, Levine FH, Daggett WM, Austen WG, Laver MB. Cardiovascular response to large doses of intravenous morphine in man. N Engl J Med 1969;281:1389-93.
5. Myles PS, Daly DJ, Djaiani G, Lee A, Cheng DC.A systematic review of the safety and effectiveness of fast-track cardiac anesthesia. Anesthesiology 2003;99:982-987.

6. Silbert B S; Myles P S. Is fast-track cardiac anesthesia now the global standard of care? Anesth Analg 2009;108:689-691.

7. Constantinides VA, Tekkis PP, Fazil A, Kaur K, Leonard R, Platt M et al. Fast-track failure after cardiac surgery: Development of a prediction model. Crit Care Med 2006;34:2875-2882.

8. Westaby S, Pillai R, Parry A, O'Regan D, Giannopoulos N, Grebenik K et al. Does modern cardiac surgery require conventional intensive care? Eur J Cardiothorac Surg 1993;7:313-318.

9. Chong JL, Pillai R, Fisher A, Grebenik C, Sinclair M, Westaby S. Cardiac surgery: Moving away from intensive care. Br Heart J 1992;68:430-433. 
10. Hawkes CA, Dhileepan S, Foxcroft D. Cochrane Review - Early extubation for adult cardiac surgical patients, 2003.

11. Van Mastrigt GA, Maessen JG, Heijmans J, Severens JL, Prins MH. Does fast-track treatment lead to a decrease of intensive care unit and hospital length of stay in coronary artery bypass patients? A meta-regression of randomized clinical trials. Crit Care Med 2006;34:1624-1634.

12. Svircevic V, Nierich AP, Moons KG, Brandon Bravo Bruinsma GJ, Kalkman CJ, van Dijk D. Fast-Track Anesthesia and Cardiac Surgery: A Retrospective Cohort Study of 7989 Patients. Anesth Analg 2009;108:727- 733.

13. Slogoff S, Keats AS. Randomised trial of primary anesthetic agents on outcome of coronary artery bypass operation. Anesthesiology 1989;70:179188.

14. Reyes A, Vega G, Blancas R, Morató B, Moreno JL, Torrecilla $\mathrm{C}$ et al. Early vs Conventional Extubation After Cardiac Surgery With Cardiopulmonary Bypass. Chest 1997;112:193201

15. Rady MY, Ryan T. Perioperative predictors of extubation failure and the effect on clinical outcome after cardiac surgery. Crit Care Med 1999;27:340-347.

16. ${ }^{16}$ Myles PS, Buckland MR, Weeks AM, Bujor MA, McRae R, Langley M et al. Hemodynamic effects, myocardial ischemia and timing of extubation with propofol- based anesthesia for cardiac surgery. Anesth Analg 1997;84:12-19.

17. Cheng DC, Karski J, Peniston C, Raveendran G, Asokumar B, Carroll J et al. Early tracheal extubation after coronary artery bypass graft surgery reduces costs and improves resource use. A prospective, randomized, controlled trial. Anesthesiology 1996:85:1300-1310.

18. Myles PS, Hunt JO, Fletcher H, Watts J, Bain D, Silvers A et al. Remifentanil, fentanyl, and cardiac surgery: A double-blinded, randomized, controlled trial of costs and outcomes. Anesth Analg 2002;95:805-812.

19. Berry PD, Thomas SD, Mahon SP, Jackson M, Fox MA, Fabri B et al. Myocardial ischaemia after coronary artery bypass grafting: Early vs late extubation. Br J Anaesth 1998;80:20-25.

20. London MJ, Shroyer ALW, Grover FL. Fast tracking into the new millennium: An evolving paradigm. Anesthesiology 1999:91:911-914.

21. Howie MB, Cheng D, Newman MF, Pierce ET, Hogue C, Hillel Z et al. A Randomized
Double-Blinded Multicenter Comparison of Remifentanil Versus Fentanyl When Combined with Isoflurane/Propofol for Early Extubation in Coronary Artery Bypass Graft Surgery. Anesth Analg 2001;92:1084-1093.

22. Cheng DC, Newman MF, Duke P, Wong DT, Finegan B, Howie $M$ et al. The Efficacy and Resource Utilization of Remifentanil and Fentanyl in Fast-Track Coronary Artery Bypass Graft Surgery: A Prospective Randomized, DoubleBlinded controlled, Multi- Center Trial. Anesth Analg 2001;92:1094-1102.

23. Möllhoff T, Herregods L, Moerman A, Blake D, MacAdams C, Demeyere $\mathrm{R}$ et al. Comparative efficacy and safety of remifentanil and fentanyl in 'fast track' coronary artery bypass graft surgery: a randomised, double blind study. BJA 2001; 87: 718-726.

24. Cheng DC, Karski J, Peniston C, Asokumar B, Raveendran G, Carroll J et al. Morbidity outcome in early versus conventional tracheal extubation after coronary artery bypass grafting: a prospective randomised control trial. J Thorac Cardiovasc surg 1996;112:755-764.

25. Flynn M, Reddy S, Shepherd W, Holmes C, Armstrong D, Lunn C et al. Fast-tracking revisited: routine cardiac surgical patients need minimal intensive care. Eur J Cardiothorac Surg 2004;25:116-122.

26. Silbert BS, Santamaria JD, O’Brien JL, Blyth CM, Kelly WJ, Molnar RR. Early Extubation Following Coronary Artery Bypass Surgery. Chest 1998;113:1481-1488.

27. Chaney MA, Furry PA, Fluder EM, Slogoff S. Intrathecal morphine for coronary artery bypass grafting and early extubation. Anesth Analg 1997;84:241-248.

28. Kogan A, Cohen J, Raanani E, Sahar G, Orlov B, Singer P. Readmission to the Intensive Care Unit After "Fast-Track" Cardiac Surgery: Risk Factors and Outcomes. Ann Thorac Surg 2003;76:503507.

29. Djaiani GN, Ali M, Heinrich L, Bruce J, Carroll $\mathrm{J}$, Karski $\mathrm{J}$ et al. Ultra-fast-track anesthetic technique facilitates operating room extubation in patients undergoing off-pump coronary revascularization surgery. J Cardiothorac Vasc Anesth. 2001;15(2):152-7.

30. Chaney M A. Intrathecal and Epidural Anesthesia and Analgesia for Cardiac Surgery. Anesth Analg 2006;102:45-64. 